\title{
Characterization of a Hypovirus-Regulated Septin Cdc11 Ortholog, CpSep1, from the Chestnut Blight Fungus Cryphonectria parasitica
}

\author{
Myeongjin Jo, ${ }^{1}$ Kum-Kang So, ${ }^{1}$ Yo-Han Ko, ${ }^{1}$ Jeesun Chun, ${ }^{1}$ Jung-Mi Kim, ${ }^{2}$ and Dae-Hyuk Kim ${ }^{1,+}$ \\ ${ }^{1}$ Department of Molecular Biology, Department of Bioactive Material Sciences, Institute for Molecular Biology and Genetics, \\ Chonbuk National University, Jeonju, Chonbuk 54896, Korea; and ${ }^{2}$ Department of Bio-Environmental Chemistry, Institute of Life \\ Science and Natural Resources, Wonkwang University, Iksan, Chonbuk 54538, Korea
}

Accepted 18 August 2018.

\begin{abstract}
We identified a protein spot showing downregulation in the presence of Cryphonectria hypovirus 1 and tannic acid supplementation as a septin subunit with the highest homology to the Aspergillus nidulans aspA gene, an ortholog of the Saccharomyces cerevisiae $\mathrm{Cdc11}$ gene. To analyze the functional role of this septin component (CpSep1), we constructed its null mutant and obtained a total of eight $\mathrm{CpSep1-null}$ mutants from 137 transformants. All CpSep1-null mutants showed retarded growth, with fewer aerial mycelia and intense pigmentation on plates of potato dextrose agar supplemented with L-methionine and biotin. When the marginal hyphae were examined, hyperbranching was observed in contrast to the wild type. The inhibition of colonial growth was partially recovered when the CpSep1-null mutants were cultured in the presence of the osmostabilizing sorbitol. Conidia production of the CpSep1null mutants was significantly increased by at least 10-fold more. Interestingly, the conidial morphology of the CpSep1null mutants changed to circular in contrast to the typical rodshaped spores of the wild type, indicating a role of septin in the spore morphology of Cryphonectria parasitica. However, no differences in the germination process were observed. Virulence assays using excised chestnut bark, stromal pustule formation on chestnut stems, and apple inoculation indicated that the CpSep1 gene is important in pathogenicity.
\end{abstract}

The chestnut blight fungus, Cryphonectria parasitica (Murrill) M. E. Barr, the causative agent of chestnut blight, has been responsible for the debilitation of chestnut forests and orchards in North America since the early 20th century (Van Alfen 1982). However, the presence of a virus with a single-stranded RNA, Cryphonectria hypovirus 1 (CHV1), attenuated fungal virulence, a phenomenon known as hypovirulence (Anagnostakis 1982;

Myeongjin Jo and Kum-Kang So contributed equally to this work.

${ }^{\dagger}$ Corresponding author: Dae-Hyuk Kim; E-mail: dhkim@jbnu.ac.kr

Funding: This work was supported by the National Research Foundation of Korea grants by MSIP (2015R1A2A1A10055684). M. Jo, K. K. So, and Y. $\mathrm{H}$. Ko were supported by the BK21 PLUS program in the Department of Bioactive Material Sciences.

*The $\boldsymbol{e}$-Xtra logo stands for "electronic extra" and indicates that four supplementary figures are published online.

(C) 2019 The American Phytopathological Society
Nuss 1992; Van Alfen et al. 1975). The C. parasitica-hypovirus system has been suggested as an ideal model system to study fungus-mycovirus interactions because many molecular tools such as reverse genetics and high-quality $C$. parasitica genome sequence information are available allowing fungal gene manipulation, and a contagious cDNA copy of the hypovirus was established for viral infection.

In our previous study, we performed proteomic and transcriptomic analyses with the supplementation of tannic acid (TA), which is found in abundance and is assumed to be one of the major phytoanticipins in the bark of chestnut trees against pathogen infection. During the proteomic analysis of C. parasitica in response to CHV1 infection or TA supplementation (Kim et al. 2012), we identified several protein spots that were differentially expressed. One differentially expressed protein spot, identified as a septin monomer, drew our attention because septin has been implicated in a myriad of functions, including not only morphogenesis and growth but also virulence.

Septins represent a conserved family of cytoskeletal GTPases that exist in the form of heteropolymeric complexes that further polymerize into filaments and have pleiotropic roles in various cellular processes such as cell division, cytoskeletal dynamics, vesicle trafficking, morphogenesis, and cell wall maintenance by serving as membrane-associated scaffolds and barriers to demarcate local compartments (Vargas-Muñiz et al. 2015; Versele and Thorner 2005). They are distributed over a wide range of eukaryotes, from animal to yeasts, excepting higher plants and certain protist (Nishihama et al. 2011; Pan et al. 2007).

Genes encoding septins were first reported in the budding yeast Saccharomyces cerevisiae, from which seven septin genes, including five mitotic septins (Cdc3, Cdc10, Cdc11, Cdc12, and Sep7/Shs1) and two sporulation-specific septins (Spr3 and Spr28), were identified. The number of septin genes varies greatly among species, from 2 in the nematode Caenorhabditis elegans to 17 in the zebrafish (Cao et al. 2007; Pan et al. 2007; Vargas-Muñiz et al. 2016).

Septins are characterized by the presence of four recognizable domains in their primary structure: the polybasic region, the GTPase domain, the septin unique element, and the coiledcoil domain (Pan et al. 2007). In yeast, it is suggested that septin monomers assemble into hetero-oligomeric complexes to further polymerize into filaments, and septin organization is tightly linked to its biological functions. Two copies of each core septin (Cdc3, Cdc10, $\mathrm{Cdc} 11$, and $\mathrm{Cdc} 12)$ in a 1:1:1:1 stoichiometry with a single Shs1 monomer were suggested to exist in hetero-oligomeric complexes (Versele and Thorner 2005; Versele et al. 2004). However, the exact mechanism governs 
the organization of septin into heteropolymeric complexes, filaments, and higher-order structures remains unknown.

Since the first discovery in yeast, the biological role of septins has been explored in diverse fungi, including the model filamentous fungi Aspergillus nidulans and Neurospora crassa, as well as pathogenic fungi infecting humans and plants (VargasMuñiz et al. 2016). Deletion mutants of the septin monomer revealed the roles of septins in morphogenesis, growth, and pathogenesis (Feng et al. 2017). However, phenotypic changes due to the mutation of each septin, even if they were orthologs, varied among fungi even when closely related. In addition, pathogenic responses of corresponding mutants were diverse, ranging from causing reduced pathogenicity to being dispensable for virulence (Kozubowski and Heitman 2010; VargasMuñiz et al. 2015, 2016; Warenda et al. 2003). In this study, we explored the biological function of a specific septin monomer, a Cdc11 ortholog, which was responsive to hypovirus infection or TA supplementation. To our knowledge, this is the first study of a host-responsive septin from a tree fungal pathogen.

\section{RESULTS}

\section{Characterization of a CpSep1 gene.}

Proteomic analysis revealed a protein spot showing accumulation changes in response to hypovirus CHV1 infection or TA supplementation. The protein spot was previously suggested to be the homolog of a septin protein (CpSEP1) (Kim et al. 2012). The corresponding gene was determined by examination of the draft genome sequence of $C$. parasitica. A 5,469-bp polymerase chain reaction (PCR) amplicon (CpSep1$\mathrm{gF} 1$ and CpSep1-gR1), which was analyzed to harbor the fulllength CpSepl gene, was cloned. Sequence analysis of the cloned PCR amplicon confirmed that the gene encoded the determined amino acid sequence. Based on the in silico analysis, a cDNA clone was acquired using reverse-transcription (RT)-PCR with the primer pair CpSep1-cF1 and CpSep1-cR1 at nucleotide positions -3 to 17 and 1,474 to 1,494 , respectively, relative to the start codon. Comparison of cDNA sequences with the corresponding genomic DNA sequences indicated that the CpSepl gene consisted of six exons, with five introns at nucleotide positions 74 to 188,306 to 364,453 to 526,665 to 718 , and 1,187 to 1,247 . All introns consisted of conserved $5^{\prime}$ GT and AG-3' junction sequences with an NCTRAC box close to the $3^{\prime}$ junction. The putative poly(A) signal (AGTAAA) was located $10 \mathrm{bp}$ downstream from the stop codon.

The deduced amino acid sequence consisted of 376 amino acids, with a molecular weight of $42.7 \mathrm{kDa}$ and a pI of 5.05 (GenBank number KY628651). Homology searches revealed that the amino acid sequence of the cloned gene was similar to other fungal septin CDC11 homologs from Diaporthe ampelina (95\% amino acid identity, KKY29390.1), D. helianthi (95\%, OCW33532.1), Valsa mali var. pyri (94\%, KUI57609.1), Pestalotiopsis fici (94\%, XP 007840562.1), Colletotrichum gloeosporioides (94\%, EQB55091.1), Eutypa lata (94\%, XP 007796174.1), C. tofieldiae (94\%, KZL78090.1), Neonectria ditissima (94\%, KPM35385.1), Microdochium bolleyi (94\%, KXJ94137.1), Fusarium oxysporum (94\%, EGU84020.1), and Hirsutella minnesotensis (93\%, KJZ75214.1). Multiple sequence alignment of 11 closely related septins with $E$ values of approximately 0.0 and a representative CDC11 from $S$. cerevisiae revealed that the cloned gene contained characteristics of conserved septin domains: the polybasic region, GTPase domain, septin unique element, and coiled-coil domain. In addition, the phylogenetic analysis using the 11 most similar septin homologs together with selected fungal septins assigned to represent core septins indicated that the cloned gene and the 11 most similar homologs branched together with septins represented by aspA of A. nidulans or $C d c 11$ of $S$. cerevisiae. The high bootstrap value of this clade suggests an apparent evolutionary relationship (Supplementary Fig. S1) (Henikoff and Hneikoff 1992). Based on the presence of the conserved septin domains and a high degree of homology to known fungal septins, we refer to the gene as CpSepl, a Cryphonectria parasitica septin gene homologous to the known fungal septin genes $C d c 11$ and $\operatorname{aspA}$.

\section{Expression of CpSep1.}

Because the proteomic analysis of the $C p S e p l$ gene exhibited downregulation in response to hypovirus infection or TA supplementation (Kim et al. 2012), the levels of transcript accumulation were examined under the corresponding conditions using quantitative real-time RT-PCR. The accumulation of CpSep1 transcripts was significantly decreased in hypovirulent UEP1 in contrast to the virus-free wild type (Fig. 1), indicating that hypovirus infection downregulated the accumulation of $C p S e p 1$ transcript in $C$. parasitica. In addition, a marked decrease of CpSepl transcript accumulation was also found in EP155/2 grown in medium supplemented with TA, which is in abundance and is assumed to be the host defense-related compound against pathogen infection. Interestingly, further decrease of the CpSepl transcript was found when the hypovirulent UEP1 strain was grown in medium supplemented with TA.

\section{Construction of a CpSep1-null mutant.}

To investigate the biological effects of mutating the CpSepl gene, we constructed a CpSepl-null mutant using split-marker deletion cassettes. Two molecular DNA cassettes, each of which contained part of the $h p h$ marker gene fused to either the 989-bp 5'-flanking or 1,087-bp 3'-flanking regions of the CpSepl gene, were used to transform protoplasts of the C. parasitica EP155/2 strain. In total, 137 single-spored transformants were screened by PCR using two primer sets, one of which was a gene-specific pair corresponding to nucleotides 625 to 650 and 1,471 to 1,511 (relative to the start codon of CpSepl,

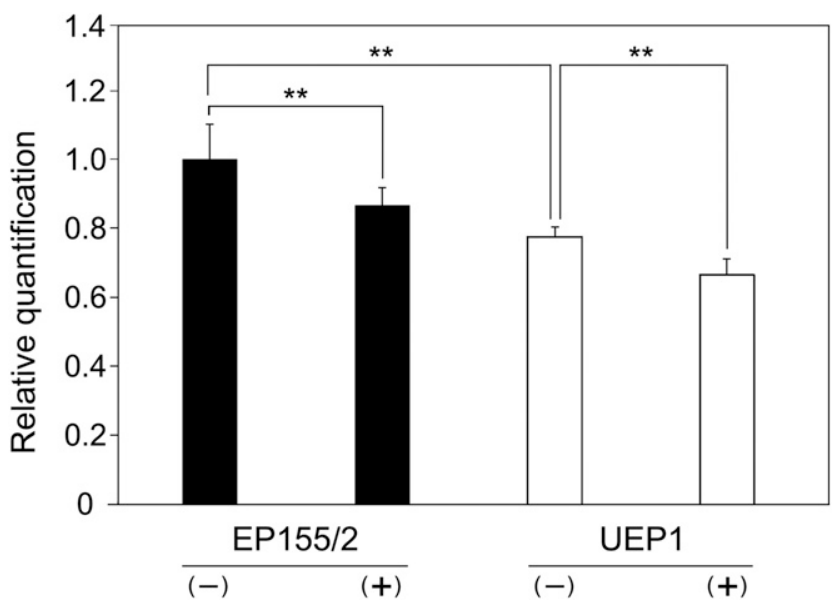

Fig. 1. Quantitative real-time reverse-transcription polymerase chain reaction analysis of $\mathrm{CpSepl}$ transcript levels in respect to levels of $\beta$-tubulin. Total RNA was prepared from the virus-free wild type (EP155/2) and its isogenic virus-containing hypovirulent strain (UEP1). Gene expression value was evaluated by the $2^{-\Delta \Delta \mathrm{CT}}$ method (Livak and Schmittgen 2001). The $y$-axis values were normalized to the C $p S e p 1$ expression in EP155/2 strain cultured $24 \mathrm{~h}$ after induction, along with standard deviations, based on the independent triplicate measurements with two independent RNA preparations of the same sample. The wild-type EP155/2 strain and its isogenic hypovirus-infected UEP1 strain are represented by closed and open bars, respectively. Symbols: + and - under the strain identification indicate with and without tannic acid supplementation, respectively, and *** indicates significant difference at the level of $P<0.01$ by paired $t$ test. 
CpSep1-gF2/CpSep1-gR2), and the other was the outer genespecific and inner $h p h$ primers corresponding to nucleotides $-1,985$ to $-1,962$ and 1,587 to 1,607 , and nucleotides 811 to 831 and 4,371 to 4,391 (relative to the start codons of CpSepl and $h p h$, respectively; Cpsep1-gf1/HPH-R1 and HPH-F2/Cpsep1gr1). Using the gene-specific primer pair, all but 21 transformants exhibited a PCR amplicon of the anticipated wild-type allele at $866 \mathrm{bp}$, while 8 transformants showed no PCR amplicon. These eight transformants were further tested by using a null-specific primer pair and all eight exhibited PCR amplicons of the expected replacement alleles of the CpSep 1 gene at 6,376 bp (data not shown). Southern blot analysis of the eight transformants was conducted to verify the gene replacement (Fig. 2). The hybridization patterns of XhoI-digested genomic DNA of the CpSepl-null mutant with a probe prepared using the 783-bp 5'-flanking region differed from the wild type and had a hybridizing band at $4.2 \mathrm{~kb}$ of the expected size of the replacement allele (Fig. 2). These results indicated that the transforming cassettes had incorporated into the CpSepl locus by site-specific integrative recombination, resulting in the replacement of $\mathrm{CpSepl}$ with part of the transforming cassettes, rather than gene disruption, as expected. Although the efficacy of gene replacement appeared to depend on various factors such as characteristics of the target gene and selection strategy, our results indicate that the split-marker approach generated an

A
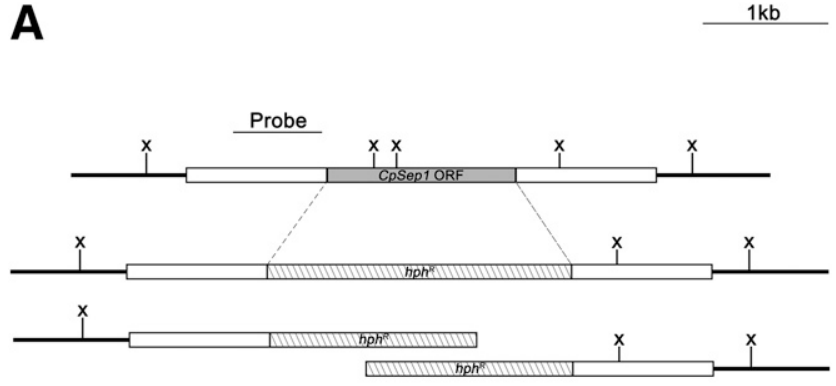

B

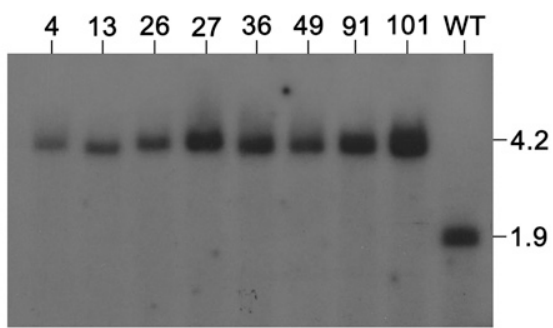

Fig. 2. Restriction enzyme map and Southern hybridization analyses of the CpSep1-null mutants and the wild-type EP155/2 strain. A, Restriction map of the CpSepl genomic region and the expected gene replacement including 989- and 1,087-bp segments as the $5^{\prime}$ - and $3^{\prime}$-flanking regions, respectively. Two molecular DNA cassettes, each of which contained part of the $h p h$ marker gene fused to either the 989-bp 5'-flanking or 1,087 bp 3'-flanking regions of CpSepl gene, are indicated under the expected gene replacement. Flanking regions and expected replacement open reading frame region are marked with open and shaded boxes, respectively. $h p h^{\mathrm{R}}$, marked with the dashed box, depicts the hygromycin B resistance cassette. Genes outside the replacement cassette are marked with lines. $\mathrm{X}$ represents restriction endonuclease XhoI. B, Southern hybridization analysis of XhoIdigested DNA from the wild-type EP155/2 strain and eight single-spored progenies of the CpSep1-null mutants (TdSEP1-4, -13, -26, -27, -36, -49, -91, and -101). The probe used is labeled on the restriction map in A and numbers on the right show the size in kilobases. The CpSepl-null mutants had undergone the derived replacement at $C p S e p 1$, as shown by the estimated changes in sizes of the bands that hybridized with the probe. WT and numbers at the top indicate the wild-type EP155/2 and eight CpSep1-null mutant strains, respectively. appropriate null mutant of $C$. parasitica and the efficacy was no less than that of a linear deletion cassette.

\section{Phenotypic characterization of the CpSep1-null mutants.}

Colony morphology of the CpSepl-null mutants differed significantly from that of the wild type (Fig. 3). Colonial growth of the CpSepl-null mutants was significantly reduced $(>50 \%)$ and mycelial growth stopped before it reached the end of the plate, resulting in irregular margins. Abnormal aerial mycelia that were shorter and less fluffy were observed at or around the center only, and few or no aerial mycelia were observed in the periphery region. Pigmentation was very intense and the tight mycelial mat was uncovered due to the absence of aerial mycelia. Because all mutant phenotypes were almost identical, we randomly selected two transformants (TdSEP1-36 and -91) for further analysis. In addition, in trans complementation of these two selected CpSepl-null mutants was carried out with a wild-type allele of the CpSepl gene to verify that the phenotypic changes were due to the replacement of the $C p S e p 1$ gene. The geneticin-resistant complemented strains containing a wild-type CpSepl gene demonstrated colony morphology comparable with that of the wild-type strain (Fig. 3). Therefore, in addition to multiple independent CpSepl-null mutant strains showing the same phenotypic changes, functional complementation using a wild-type $C p S e p l$ gene clearly indicated that the mutation in the CpSepl gene caused the morphological changes in the mutant strains.

Because the colony margin and aerial mycelia appeared abnormal, we examined the branching pattern of marginal hyphae. The wild type exhibited a regular branching pattern, with hyphae diverging from one another at the growing tip, whereas the CpSep1-null mutant showed a more intense hyphal network due to the hyperbranching pattern (Fig. 4).

Cell wall integrity was examined using cell-wall-disturbing agents. When the CpSepl-null mutant and the wild type were grown on medium containing cell-wall-disturbing agents, no significant change was observed in response to the agents (Supplementary Fig. S2). Responses to temperature stress were examined at diverse temperatures; no considerable changes in colony morphology were observed (data not shown). In addition, responses against oxidative stress in the CpSepl-null mutants were also examined by comparing colony morphology in the presence or absence of menadione; a meaningful increase in sensitivity was observed in the CpSepl-null mutants (Fig. 5). These results suggest that responses to stresses, including cellwall inhibitors, temperature, and oxidative stress, varied with respect to the mutation of the CpSepl gene. We tested whether septin is important in responses to osmotic changes by growing the CpSep1-null mutant on potato dextrose agar supplemented with L-methionine and biotin (PDAmb) supplemented with various concentrations of sorbitol (Fig. 6). The colonial growth of the CpSepl-null mutant recovered on PDAmb supplemented with concentrations of sorbitol up to $1.5 \mathrm{M}$. Then, the growth of the mutant was severely retarded at $2.0 \mathrm{M}$ sorbitol, the concentration at which the wild type was also severely inhibited. Thus, supplementation of low and medium concentrations of sorbitol restored the growth of the mutant but high concentrations of sorbitol limited the growth of fungus, whether mutant or wild type.

\section{Conidiation, spore characteristics, and germination.}

We examined the spore production of the CpSepl-null mutant. When we measured the number of conidia produced per PDAmb plate, conidia production was markedly increased in the CpSepl-null mutant compared with that of the wild type. Ten-fold more conidia were produced in the CpSepl-null mutant (Table 1). Considering the restricted colonial growth of the 
CpSep1-null mutant, conidia production per unit area of mycelia appeared to be even greater than 10-fold. As far as we know, this is the first report of increased conidia production due to septin mutation.

Spore morphology was greatly changed. The conidia of the CpSep1-null mutant were more round and spherical than those of the wild type and complemented strains (i.e., they changed from the typical straight or slightly curved-oblong to cylindrical shape into a subglobose to round shape) (Fig. 7). Because we observed that osmotic stability was important for appropriate growth in this mutant and that spore shape might be affected during the preparation of conidia suspension, scanning electron microscopy (SEM) was performed on conidia in the conidia-bearing structure. SEM confirmed that excess conidia were present in each fruiting structure of the CpSepl-null mutant and conidia were more round and spherical than in the wild type (Supplementary Fig. S3).

Spore germination was observed. Most of the cylindricalshaped conidia of the wild type became swollen after a $12-\mathrm{h}$ incubation and these swollen spores also produced young germ tubes at $18 \mathrm{~h}$ postincubation (hpi) (Supplementary Fig. S4). Then, young germlings started to show extensive polar growth and distinctive septations. Most conidia germinated from one side but the second germination at the opposite side of the prior germination site was not uncommon ( $\geq 24 \mathrm{hpi})$. For the CpSeplnull mutant, the swelling and germination followed by extensive polar growth were comparable with the wild type. Compared with the wild type, no significant change in the germination rate of the conidia of the CpSepl-null mutant was observed. In addition, once germinated, septations were normal.

Because the cytoskeleton is important for maintaining cell shape (Banuett and Herskowitz 2002; Brunner and Nurse 2000), we visualized the actin cytoskeleton in spores and young hyphae using a specific antibody. Although weak uniform fluorescence was observed over the entire cell surface, intense fluorescence was observed in a bulge in spores and young hyphae, indicative of an actin patch. In the wild-type conidia, densely stained actin patches were observed in the protrusion at the apex of swollen cylindrical conidia and at the tip of emerging germ tubes, which suggested the concentration of a multivesicle cluster at the tip of the emerging germ tube and the secretion of cell wall components for the initial polar growth (Fig. 8). In addition, apical fluorescent patches were observed at the tips of the actively growing young hyphae. No dramatic changes in the localized distribution of actin patches at the emergence of germ tube were observed in the swollen spherical conidia of the CpSep1-null mutant (Fig. 8). However, marked changes in the location of actin patches were observed in the growing hyphae of the CpSepl-null mutant (i.e., subapical instead of apical localization of actin patches was observed some distance behind the hyphal tip) (Fig. 8).

\section{Virulence assay.}

A virulence assay performed on the excised chestnut tree bark revealed that the TdSEP1-36 and -91 mutants produced smaller-sized necrotic areas than the wild-type EP155/2 (Fig. 9A). Additionally, the sizes of the necrotic areas were even smaller than those of the hypovirulent UEP1 strain. No significant differences in the necrotic area were observed between the wild type and two complemented strains, as expected (Fig. 9A). Considering that the CpSepl-null mutants showed significantly reduced growth, these smaller-sized necrotic areas might be attributable to the growth defect. Therefore, we examined whether disruption of the CpSepl gene affected the fungal pathogenic growth on the tree by observing the stromal pustule eruption on chestnut stems, as previously described (Kazmierczak et al. 2005). Mutants TdSEP1-36 and -91 showed significantly fewer eminent stromata than the wild type and

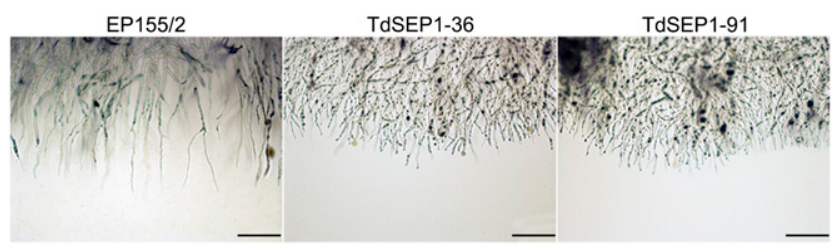

Fig. 4. Microscopic observation of the branching pattern of marginal hyphae of the CpSep1-null mutant strains (TdSEP1-36 and -91). Note that the peripheral hyphae appear more intense due to the hyperbranching pattern in the $C p S e p 1$-null mutants. Strains indicated at the left are the wild-type EP155/2 and CpSep1-null mutant (TdSEP1-36 and -91) strains. Scale bars indicate $200 \mu \mathrm{m}$.

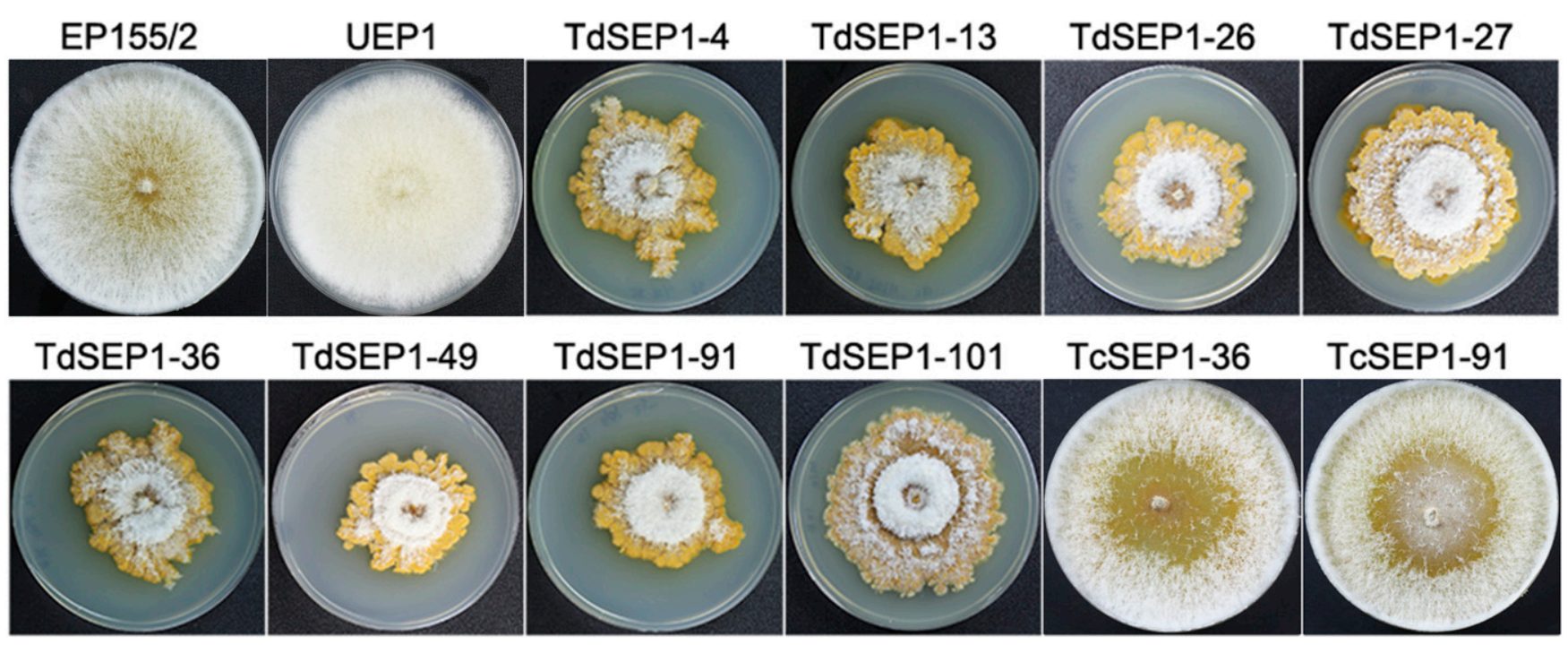

Fig. 3. Colony morphology of the CpSep1-null mutant strain. Colonies after 14 days of culturing on potato dextrose agar supplemented with L-methionine and biotin are shown. Strains indicated below the panel are the wild-type EP155/2, hypovirulent UEP1, eight independently isolated $C p S e p 1$-null mutant strains (TdSEP1-4, -13, -26, -27, -36, -49, -91, and -101), and two complemented strains (TcSEP1-36 and TcSEP1-91). Note that, among these CpSep1-null mutant strains, two representative mutants, TdSEP1-36 and TdSEP1-91, were selected for further analyses, and corresponding complemented strains of TdSEP1-36 (TcSEP1-36) and -91 (TcSEP1-91) are shown as comparisons. 
complemented strains, suggesting that the CpSep1-null mutants had constraint in efficient colonization and growth under the bark of chestnut twigs (Fig. 9B). In addition, we also performed an apple assay (Kim et al. 2001). The CpSepl-null mutant showed a dramatically reduced $(<20 \%)$ brown-colored region (Fig. 9C), suggesting that the CpSep1-null mutants were less virulent than the wild type and complemented strains. Although the growth defect of the CpSep1-null mutants contributed to the reduced virulence, the marked decrease of eminent stromata on chestnut stems and the remarkably reduced brown-colored lesion area strongly suggest that the $C p S e p l$ gene plays a part in the virulence of this fungus.

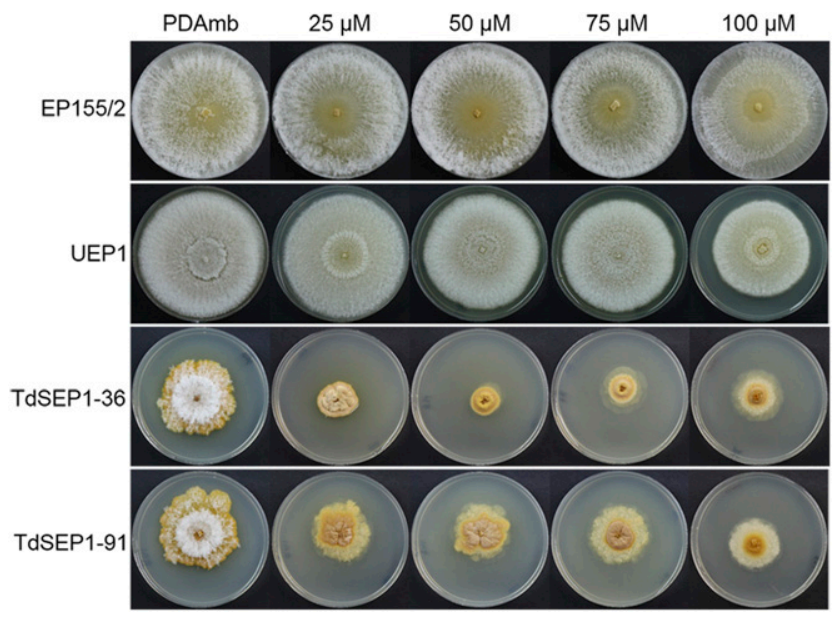

Fig. 5. Effect of oxidative stress on fungal growth. Menadione, an $\mathrm{H}_{2} \mathrm{O}_{2}$ producer, was added to the potato dextrose agar supplemented with $\mathrm{L}$ methionine and biotin (PDAmb) medium to induce oxidative stress. Colonies after 10 days of culture on PDAmb are shown. Note that the radial growth of the CpSep1-null mutants (TdSEP1-36 and TdSEP1-91) was severely retarded compared with that of the wild type.

\section{DISCUSSION}

The CpSepl-null mutant in this study showed restricted colonial growth and irregular hyperbranching at the colony margin. These results suggest that radial extension of the mutant colony was retarded, partly due to hyperbranching. Thus, the CpSepl gene is involved in preventing the inappropriate emergence of branches in order to maintain polar growth. These findings were in good agreement with the results of immunofluorescent staining of the actin cytoskeleton, which demonstrated the subapical localization of actin patches in the actively growing young hyphae of the CpSepl-null mutant. Densely stained actin patches at the tip of growing hyphae indicate the presence of an apical vesicle cluster that maintains the polarized growth of hyphae. Consequently, subapical instead of apical actin patches suggested the incorporation of new wall material at this site and the occurrence of premature polar growth, which resulted in hyperbranching of the CpSepl-null mutant at the colony margin. Although we did not measure the fusion capacity, the rareness of lateral hyphae interconnecting with the adjacent hyphae suggests that the fusion capacity of the CpSepl-null mutant was impaired. This might explain the reduced aerial mycelia, which require intense networking among hyphae for physical support.

Our mutant showed restoration of mycelial growth under the presence of intermediate ranges of osmotic stabilizer but, at a higher concentration of sorbitol $(2.0 \mathrm{M})$, reflecting high osmotic stress, it showed limited growth, as expected, indicating

Table 1. Conidia production of null mutants compared with the wild type

\begin{tabular}{lll}
\hline Strain & \multicolumn{1}{c}{ Characteristic } & Number of conidiospores/ml \\
\hline EP155/2 & Wild type & $4.83 \times 10^{7} \pm 3.06 \times 10^{6}$ \\
TdSEP1-36 & CpSepl-null mutant & $4.30 \times 10^{8} \pm 3.00 \times 10^{7 * *}$ \\
TdSEP1-91 & CpSep1-null mutant & $4.57 \times 10^{8} \pm 6.11 \times 10^{7 * *}$ \\
\hline
\end{tabular}

a Asterisks (**) indicate Student's $t$ test significant at $P<0.01$.

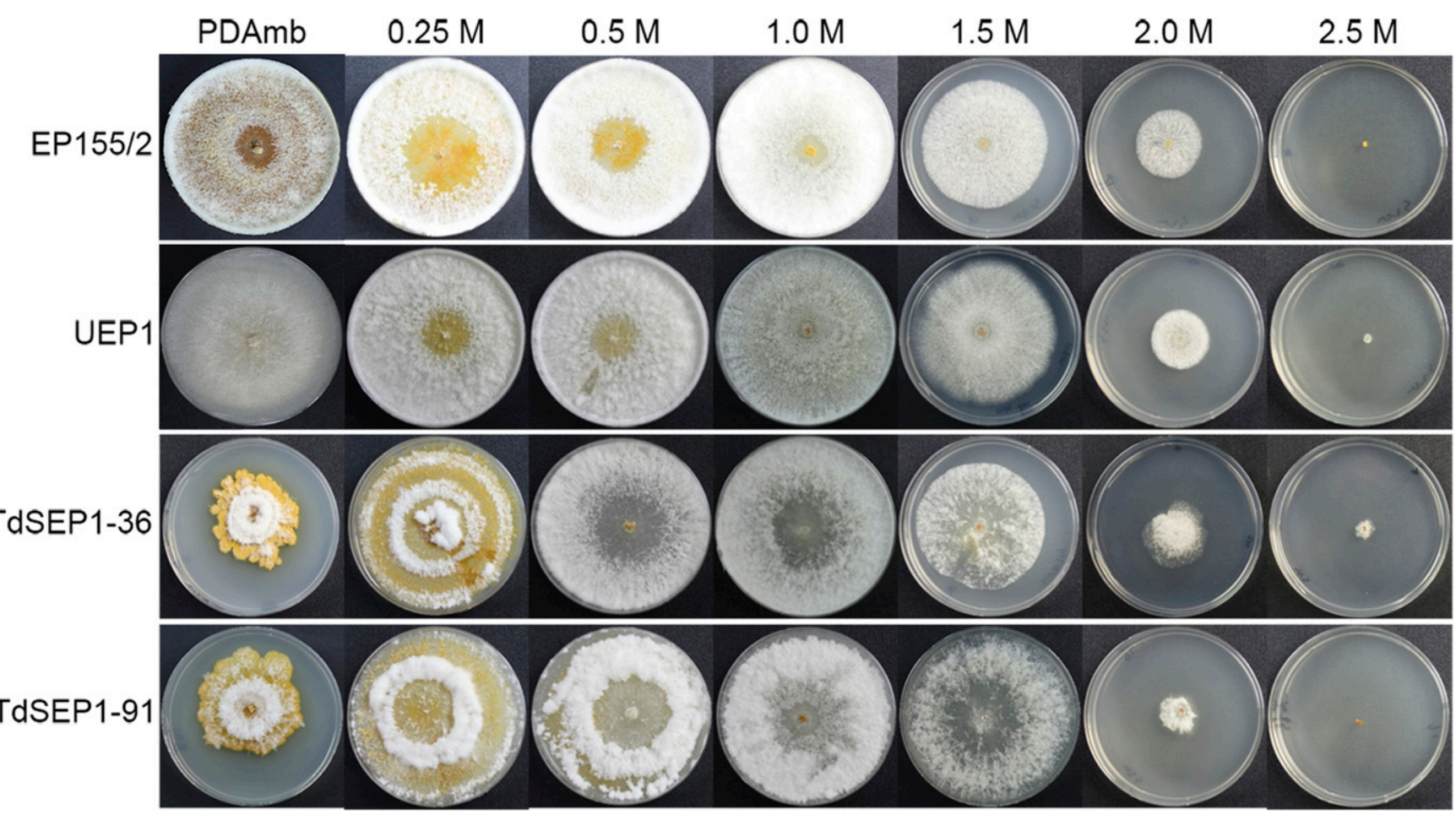

Fig. 6. Conidial morphology of the CpSep1-null mutant strains in response to the osmotic stabilizer. Colonies after 10 days of culture on potato dextrose agar supplemented with L-methionine and biotin (PDAmb) with the addition of sorbitol are shown. Strains shown at the left are the wild-type EP155/2, hypovirulent UEP1, and two representative CpSep1-null mutant strains (TdSEP1-36 and -91). Numbers at the top of the panel indicate the concentration of sorbitol. 
that the growth rate was proportionally lower than in the wild type. In several studies, mutants related to cell wall integrity have shown partial growth recovery after supplementation with an osmotic stabilizer (Borgia et al. 1996; Fillinger et al. 2001; Li et al. 2006). However, the CpSepl-null mutant in this study did not show any remarkable changes against cell-walldisturbing agents. These results suggest that the protein product of the CpSepl gene is involved in osmoregulation within the hyphae in relation to proper septation rather than in cell wall construction.

Defective or reduced conidiation, as well as no effect on conidiation, are common phenotypes of septin mutants. Two different roles of septin have been implicated in conidiation: defects in the separation between proconidia followed by their separation into spores in Neurospora crassa (Berepiki and Read 2013) and defects in the production of the specialized sporeforming structures of A. nidulans and Cryptococcus neoformans (Hernández-Rodríguez et al. 2012; Kozubowski and Heitman 2010). However, the CpSepl-null mutant showed significantly increased conidiation. No significant changes in the spore-bearing structures of Cryphonectria parasitica were observed using SEM. These results propose that the CpSepl gene may have a function in modulating new spore formation, which might be similar to suppressing new growth (HernándezRodríguez et al. 2012). Moreover, we found marked morphological changes in the conidia from the canonical oblong to cylindrical shape into a subglobose to round shape. Although changes from elliptical to more globose were also observed in
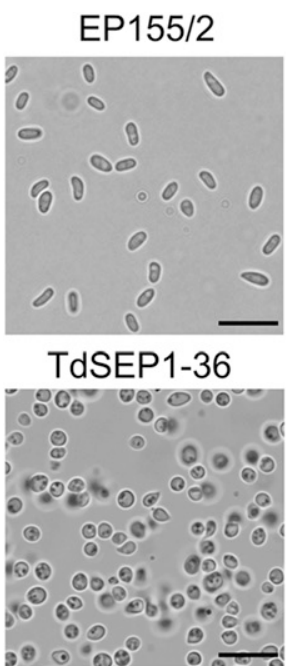

TdSEP1-4

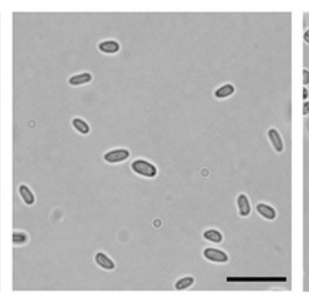

TdSEP1-49

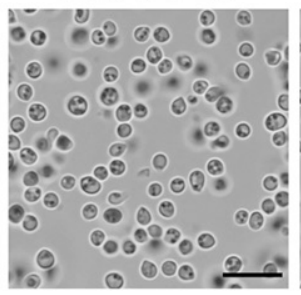

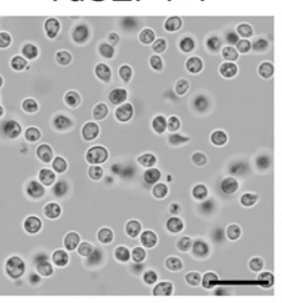

TdSEP1-91

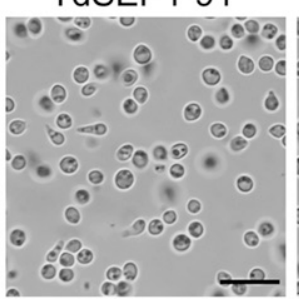

TdSEP1-13

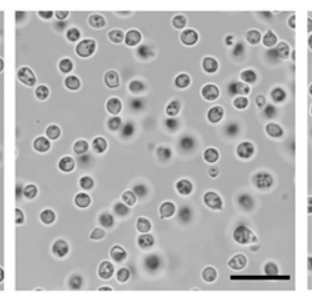

TdSEP1-101

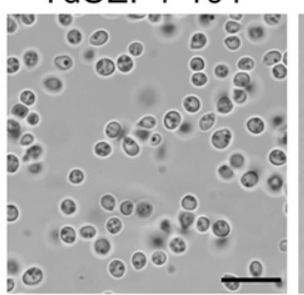

TdSEP1-26

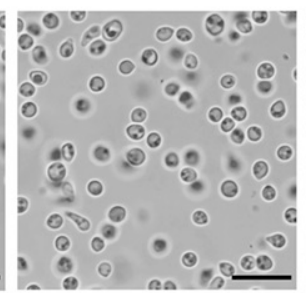

TCSEP1-36

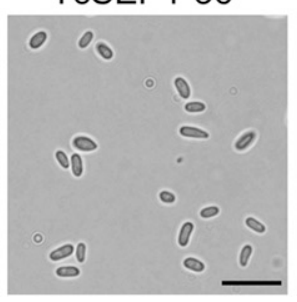

TdSEP1-27

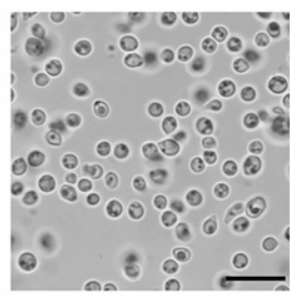

TcSEP1-91

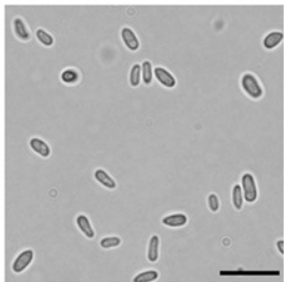

Fig. 7. Conidial morphology of the CpSep1-null mutants. Conidia were obtained from 4-week-old potato dextrose agar supplemented with L-methionine and biotin plates and observed under the microscope. Strains indicated below the panel are the wild-type EP155/2, hypovirulent UEP1, eight independently isolated CpSep1-null mutant strains (TdSEP1-4, -13, -26, -27, -36, -49, -91, and -101), and two complemented strains (TcSEP1-36 and TcSEP1-91). Scale bars indicate $10 \mu \mathrm{m}$.

\section{EP155/2}

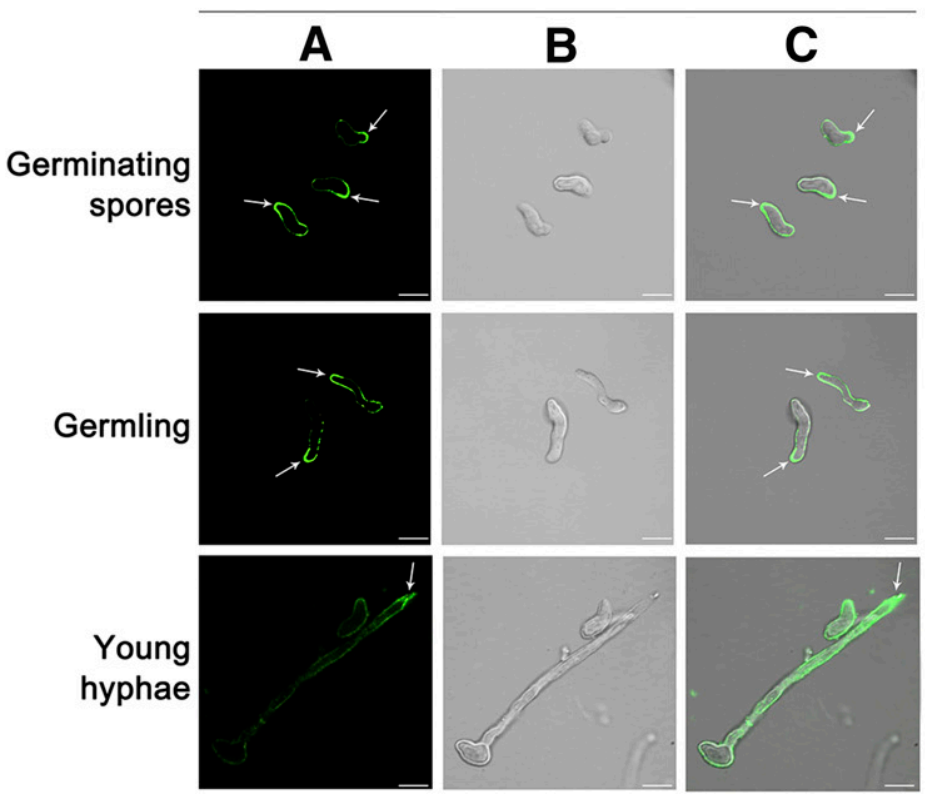

TdSEP1-36

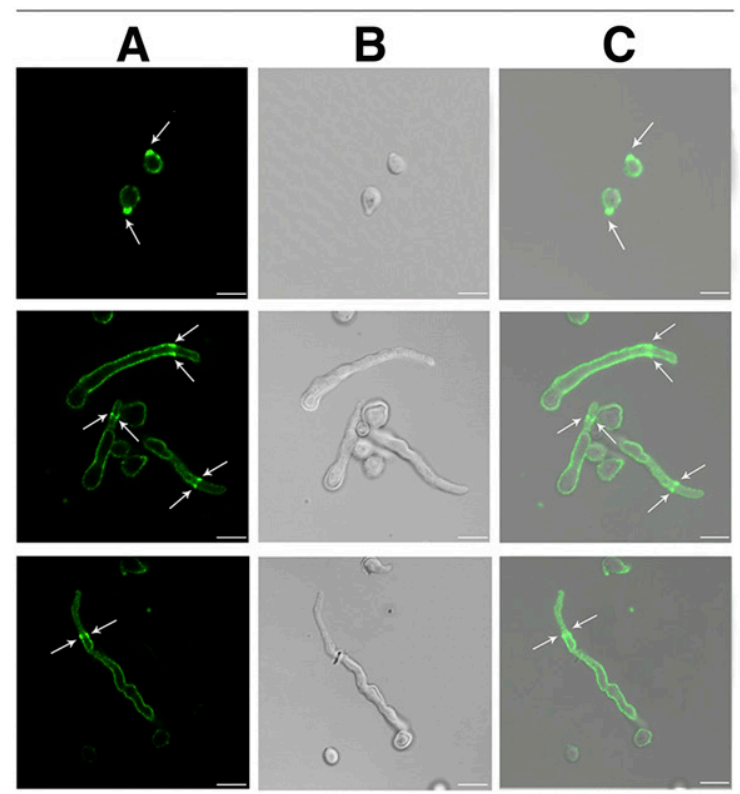

Fig. 8. Organization of the actin cytoskeleton in germinating spores, young germlings, and actively growing young hyphae. Column A, Immunofluorescence using an antiactin antibody to visualize actin in spores and young hyphae; column B, light microscopy images of the corresponding immunofluorescent images; and column C, a merged image of A and B. Strains and samples are indicated at the top and on the left, respectively. Arrows point to the densely stained actin patches. Scale bars in all panels indicate $5 \mu \mathrm{m}$. 
Botrytis cinerea, F. graminearum, and Ustilago maydis (Alvarez-Tabarés and Pérez-Martín 2010; Chen et al. 2016; Feng et al. 2017), the conidial morphogenetic changes were more dramatic in the CpSepl-null mutant, suggesting that, in conidia, the CpSepl gene plays a role in scaffolds for proteins involved in spore morphology. The germination ratio of the mutant conidia being similar to that of wild type indicates that the conidia of the CpSepl-null mutant had no viability defect.

A
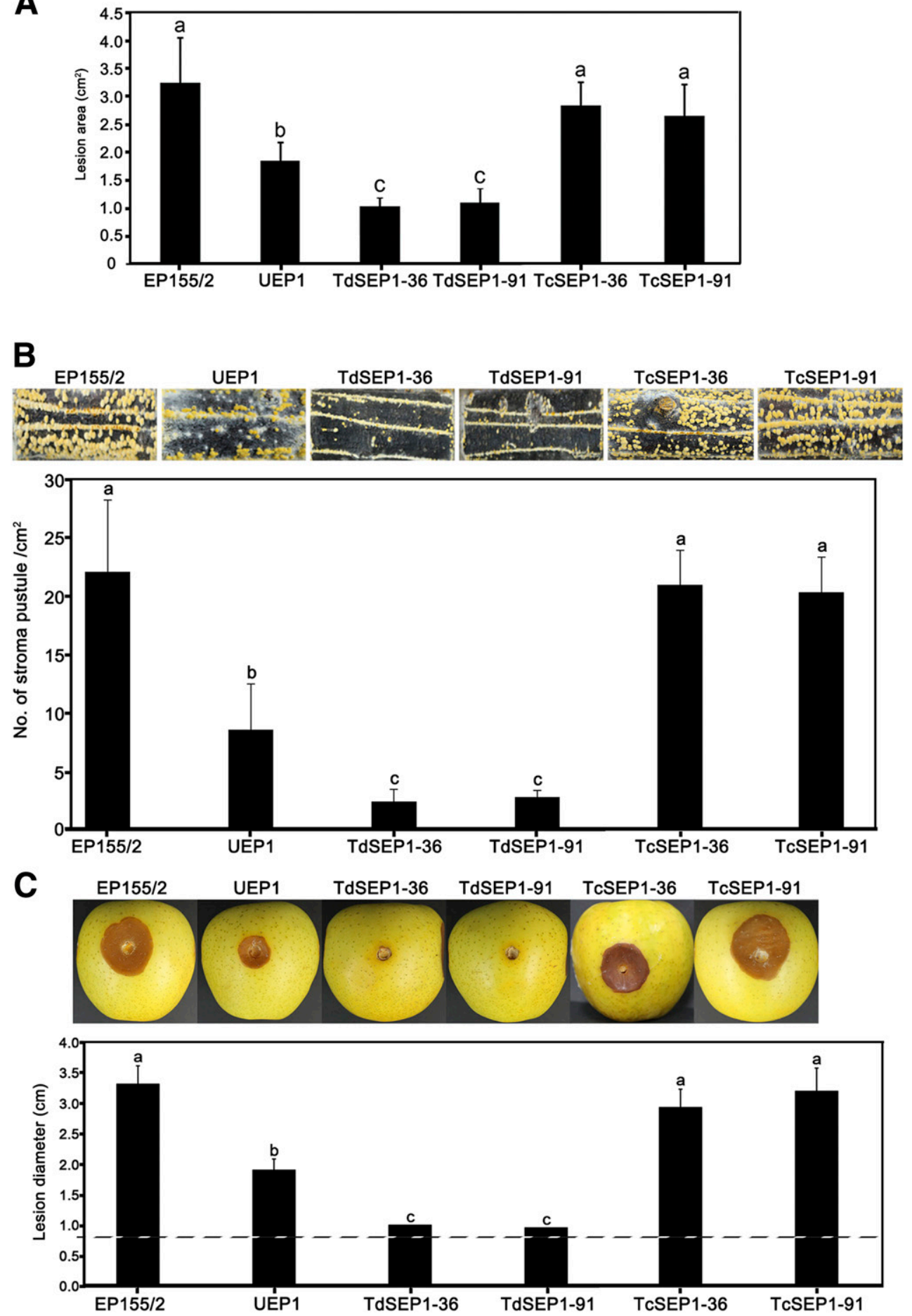

Fig. 9. Virulence assays. A, Virulence assay on excised chestnut tree bark performed as described previously (Lee et al. 1992). Lesion values of the measurements are represented as means \pm standard deviation (square centimeters). Triplicates for each strain were applied, and experiments were performed in duplicate. Different letters imply significant differences between treatments based on Duncan's multiple range test at $P=0.05$. B, Fungal invasive growth on the chestnut tree stem examined as described previously (Kazmierczak et al. 2005). Chestnut stems were artificially wounded with three cuts using a razor blade and inoculated on each side of stem by placing three agar blocks containing actively growing mycelia. Note that both wounded and unwounded areas showed markedly reduced numbers of distinctive stromal pustules. C, Virulence assay using apple inoculation as described previously (Fulbright 1984). A representative image of apple inoculation 10 days after inoculation on Hwang-Ok apple fruit is shown. Lesion diameter values are shown in the lower panel as means \pm standard deviation (centimeters). The dashed line indicates diameter induced by using a $0.8-\mathrm{cm}$ cork borer to punch holes in an apple for inoculation. Strains used were the wild-type EP155/2, hypovirulent UEP1, CpSep1-null mutants (TdSEP1-36 and -91), and two complemented strains (TcSEP1-36 and TcSEP1-91). Five replicates for each strain were used for analysis, and each experiment was performed in duplicate. Different letters imply significant differences between treatments based on Duncan's multiple range test at $P=0.01$. 
A. fumigatus exhibited changes in spore morphology due to the absence of the electron-dense outer conidial wall in the aspB-null mutant strain (Vargas-Muñiz et al. 2015). However, in the CpSepl-null mutant, there were no changes in the germination ratio in the presence of cell-wall-disturbing agents, suggesting no significant changes in the wall organization of the conidia. These results suggest that changes in the conidial morphology of the CpSepl-null mutant were not indirectly attributable to defects in the conidial wall architecture; however, the protein products of the CpSepl gene are implicated in determining the conidial morphology, probably as a cytoskeletal component. It is interesting that the protein product and transcription of the CpSepl gene were significantly downregulated as a response to hypovirus whereas loss-of-function mutation of the CpSepl gene demonstrated significantly increased production of conidia with marked morphological changes. These results suggest that the CpSepl gene may be implicated in the conidiation by the process of quality check, which requires further studies.

Bioinformatic and phylogenetic analyses of the draft genome sequence of $C$. parasitica suggest the presence of another three orthologs of core septin monomers (orthologs to A. nidulans AspB, AspC, and AspD or S. cerevisiae Cdc3, Cdc12, and Cdc10) and an ancestral septin ortholog (AspE), which is common in filamentous ascomycetes (Momany et al. 2001; Pan et al. 2007). However, not all septin monomers responded to the hypovirus infection and TA supplementation; only the protein product of the CPSepl gene, an ortholog of aspA and Cdc11, was influenced by the hypovirus infection and TA supplementation. These results suggested that a specific, not general, component in the septin complex is the target of hypovirus infection and TA supplementation. As suggested in other filamentous fungi (Berepiki and Read 2013; Juvvadi et al. 2011; Vargas-Muñiz et al. 2016), an individual septin monomer may perform different roles in morphogenesis; for example, in A. fumigatus, the deletion of aspD exclusively led to defective conidiation and the $a s p E$ mutation only influenced septation but not conidiation (Vargas-Muñiz et al. 2015). In addition, even orthologous septins have functional diversity among different fungi; that is, the effects of orthologous septin monomers on virulence, cell wall formation, and septation varied among different fungi (Berepiki and Read 2013; Feng et al. 2017; Vargas-Muñiz et al. 2015). Thus, the $C p S e p 1$ gene product may have a function unique from other septin monomers during the hypovirus infection and host plant invasion. One possible role of the CpSepl gene in response to viral infection and TA supplementation is that decreased accumulation of the CpSepl gene product as a result of viral infection, and TA supplementation limits the fungus to maintain appropriate fungal growth and pathogenicity because the CpSepl gene is implicated in normal polar growth with appropriate branching. Interestingly, the fact that downregulation of the CpSepl gene transcript was severed when viral infection and TA supplementation were present together suggests that two different regulatory pathways exist. In addition, this is the first report of a septin mutation resulting in enhanced, not reduced, conidia production. Although the septin mutant of $S$. cerevisiae produced a multibud phenotype, it was speculated that this resulted from incomplete cytokinesis separating buds from mother cells, not from the concurrent appearance of daughter cells (Longtine et al. 2000). Similarly, impaired conidiation of septin mutants of filamentous fungi was ascribed to cell division or separation defects (Feng et al. 2017; Lindsey et al. 2010; Vargas-Muñiz et al. 2016). However, this study suggests that the CpSepl gene plays roles in the modulation of conidia production. Based on the increased conidia production by the CpSep1-null mutant, downregulation of the CpSEP1 protein in response to hypoviral infection may result in sustaining conidia production upon hypovirus infection.

In conclusion, the CpSepl-null mutant exhibited reduced growth, aberrant hyperbranching, increased conidia production with dramatic changes in conidia shape, no changes in cell wall stress response but hypersensitive to reactive oxygen species and recovered growth upon supplementation with an osmotic stabilizer, restricted growth in the host, and reduced virulence. Among these, increased conidiation, dramatic changes in conidial shape, and recovery upon osmotic supplementation in the absence of a defect in response to cell wall stress have not been observed in other fungi.

\section{MATERIALS AND METHODS}

Fungal strains and growth.

C. parasitica strain EP155/2 (ATCC 38755), its isogenic CHV1-713 with hypovirulent strain UEP1, and the resulting transformants were maintained and cultured as described previously (Kim et al. 1995). PDAmb and complete media were used as the basic media for plate and liquid cultures, respectively, of the fungus (Puhalla and Anagnostakis 1971). TA induction of fungal strains was assessed in accordance with the protocol described previously (Kim et al. 2012). Measurement of radial growth and conidial quantification were performed as described previously (Kim et al. 1995). The mycelia were harvested and lyophilized until use as described previously (Powell and Van Alfen 1987).

\section{Cloning and characterization of a Cdc11-like gene, CpSep1.}

Tandem mass spectrometric analysis of a selected protein spot followed by inspection of the genome database of $C$. parasitica identified the gene encoding the determined amino acid sequence. The selected protein spot corresponded to an ortholog of the $S$. cerevisiae $C d c 11$ gene. PCR amplification of this gene was performed using primers CpSep1-gF1 and CpSep1-gR1; the resulting 5,469-bp PCR product clone was inserted into the pGEM-T Easy vector and sequenced.

To get the nearly full-length cDNA clone of CpSep1, in silico analysis of the genomic DNA sequence was performed and RT-PCR was conducted with primers CpSep1-cF1 and CpSep1-cR1. The resulting 1,134-bp cDNA amplicon was

Table 2. Primers used in this study

\begin{tabular}{ll}
\hline Primer & \multicolumn{1}{c}{ Sequence $\left(\mathbf{5}^{\prime}-\mathbf{3}^{\prime}\right)$} \\
\hline CpSep1-gF1 & CCATAAGAAGATCGCCCGTACGT \\
CpSep1-gR1 & ACGCGGTACCTGCAGGTGGT \\
CpSep1-gF2 & TCATGCCATGCTCTACTTCATTACA \\
CpSep1-gR2 & AAAAGTTCTTCTCCTTTACTAGCAGCTTCCGTC \\
& TCGCCGT \\
CpSep1-cF1 & AAGATGATCCGCCGCAAGAA \\
CpSep1-cR1 & TTAAGCAGCTTCCGTCTCGC \\
Sep1-F1 & GGAGTGAACTCAGTTGCAATGC \\
Sep1-R1 & TCCTTCAATATCATCTTCTGTCGACTAGAATCG \\
& TTGCATGTCAGCAG \\
Sep1-F2 & GCATAAGGGAGAGCGTCGACAGGCCTGGAGTAA \\
& ATGTGGC \\
Sep1-R2 & AGCTGCGATCAGCTTGTACCT \\
Hph-F1 & CTGCTGACATGCAACGATTCTAGTCGACAGAAG \\
& ATGATATTGAAGGA \\
Hph-R1 & ACCCGAAAACGCGTTTTATT \\
Hph-F2 & ATTTCATATGCGCGATTGCT \\
Hph-R2 & GCCACATTTACTCCAGGCCTGTCGACGCTCTCCC \\
& TTATGC \\
RT-tub-F & GTCATTACACCGAGGGTGCT \\
RT-tub-R & TACCAGCACCAGTACCACCA \\
RT-Sep1-F & CGCATCCCCGTATACAACTT \\
RT-Sep1-R & ATCTCGACCGTCTCCTCAGA \\
\hline
\end{tabular}


cloned and sequenced. All primers used in this study are listed in Table 2.

\section{Southern blot analyses.}

Genomic DNA extraction from C. parasitica and Southern blot analysis using the restriction enzyme XhoI were conducted as previously described (Churchill et al. 1990).

\section{Analysis of transcript accumulation using quantitative real-time RT-PCR.}

To evaluate the expression levels of target genes, quantitative RT-PCR was conducted as previously described (Park et al. 2012). Each expression level was evaluated in triplicate for each transcript, with at least two independent preparations of the same RNA sample. Primer pairs for $\beta$-tubulin and CpSep1 genes are indicated as RT-tub-F/RT-tub-R and RT-Sep1-F/RTSep1-R, respectively (Table 2). The abundance of CpSep1 transcript, relative to the amount of $\beta$-tubulin gene, in the sample was estimated using the $2^{-\Delta \Delta C T}$ method as described previously (Livak and Schmittgen 2001).

\section{Targeted gene replacement and fungal transformation.}

Targeted gene replacement using split-marker deletion cassettes was applied to examine the biological effects of the CpSepl gene (Goswami 2012). Two molecular DNA cassettes, each of which contained a part of the hygromycin B phosphotransferase gene cassette $(h p h)$ fused to either the 2,596-bp $5^{\prime}$-flanking or 2,683-bp 3'-flanking regions of the CpSepl gene, were prepared by overlap PCR (Sheppard et al. 2005) as follows. A 1,000-bp PCR amplicon containing the 989-bp 5'flanking region of $C p S e p l$ was amplified using gene specific primers Sep1-F1 and Sep1-R1. A 1,629-bp part of a selection marker gene containing the promoter and part of the $\mathrm{N}$ terminus was amplified using primers Hph-F1 and Hph-R1. Fusion of these two PCR amplicons was conducted using overlap extension PCR with primers Sep1-F1 and Hph-R1. A 2,683-bp PCR amplification of the 1,087-bp 3'-flanking region and the 1,596-bp part of the selection marker gene containing the terminator and part of the $\mathrm{C}$ terminus was amplified and fused using primers Sep1-F2/Sep1-R2, Hph-F2/Hph-R2, and HphF2/Sep1-R2. The resulting molecular cassettes were then used simultaneously to transform protoplasts of the $C$. parasitica EP155/2 strain.

Complementation analysis of the CpSepl-null mutant was assessed by transforming the mutant with the wild-type allele. The complementing vector $\mathrm{pCSep} 1$ was constructed by ligation of a 4,611-bp NotI and XbaI fragment of pBluescript SK(-) containing the geneticin resistance cassette (Nguyen et al. 2008), with NotI- and SpeI-digested pCSep1 carrying a 5,491bp fragment with the full-length $C p S e p 1$ gene. The ligated plasmid was then used for transformation of the putative CpSep1-null mutant.

Preparation and transformation of protoplast were conducted as described previously (Churchill et al. 1990; Kim et al. 1995). Selection of transformants via successive transfer on the selection medium with the addition of hygromycin B at $150 \mu \mathrm{g} / \mathrm{ml}$ (Calbiochem) or geneticin at $150 \mu \mathrm{g} / \mathrm{ml}$ (Invitrogen) and singlespore isolation were performed as previously described (Kim et al. 2004; Nguyen et al. 2008). To verify replacement and in trans complementation of the CpSepl gene, PCR and Southern hybridization were performed.

\section{SEM and fluorescent microscopy.}

To evaluate the conidia and conidia-bearing structures of the $C$. parasitica strains using SEM, preparation of fungal mycelia and treatment of mycelial fragments were carried out as previously described (Kim et al. 2016). The specimens were observed as previously described (Kim et al. 2004).

To visualize the actin cytoskeleton, indirect immunofluorescence using a monoclonal antiactin antibody produced in a mouse (Sigma-Aldrich) and antimouse immunoglobulin G fluorescein isothiocyanate conjugate (Sigma-Aldrich) were used as the primary and secondary antibodies, respectively, and performed as previously described (Banuett and Herskowitz 2002). Briefly, freshly harvested conidia of $C$. parasitica were suspended in EP complete media and incubated on a coverslip that had been pretreated with $0.01 \%$ poly-L-lysine (SigmaAldrich). To digest the cell walls, the conidia and young hyphae were fixed with $3.7 \%$ formaldehyde, treated with Glucanex (Novozymes) $(5 \mathrm{mg} / \mathrm{ml}$ in $50 \mathrm{mM}$ sodium citrate, $1 \mathrm{M}$ sorbitol, and $5 \mathrm{mM}$ EGTA), and stained with the antiactin antibody. After staining, the cells were observed using a confocal laserscanning microscope (LSM 880 model; Carl Zeiss).

\section{Statistical analysis.}

The number of conidia and canker areas were analyzed by Student's $t$ test or analysis of variance using IBM SPSS version 23 , and the significant effects on the strains and treatments were evaluated by Duncan's multiple-range test at $P=0.05$ and 0.01 .

\section{LITERATURE CITED}

Alvarez-Tabarés, I., and Pérez-Martín, J. 2010. Septins from the phytopathogenic fungus Ustilago maydis are required for proper morphogenesis but dispensable for virulence. PLoS One 5:e12933.

Anagnostakis, S. L. 1982. Biological control of chestnut blight. Science 215:466-471.

Banuett, F., and Herskowitz, I. 2002. Bud morphogenesis and the actin and microtubule cytoskeletons during budding in the corn smut fungus, Ustilago maydis. Fungal Genet. Biol. 37:149-170.

Berepiki, A., and Read, N. D. 2013. Septins are important for cell polarity, septation and asexual spore formation in Neurospora crassa and show different patterns of localisation at germ tube tips. PLoS One 8: e63843.

Borgia, P. T., Miao, Y., and Dodge, C. L. 1996. The orlA gene from Aspergillus nidulans encodes a trehalose-6-phosphate phosphatase necessary for normal growth and chitin synthesis at elevated temperatures. Mol. Microbiol. 20:1287-1296.

Brunner, D., and Nurse, P. 2000. New concepts in fission yeast morphogenesis. Philos. Trans. R. Soc. Lond. B Biol. Sci. 355:873-877.

Cao, L., Ding, X., Yu, W., Yang, X., Shen, S., and Yu, L. 2007. Phylogenetic and evolutionary analysis of the septin protein family in metazoan. FEBS Lett. 581:5526-5532.

Chen, S. N., Luo, C. X., Hu, M. J., and Schnabel, G. 2016. Fitness and competitive ability of Botrytis cinerea isolates with resistance to multiple chemical classes of fungicides. Phytopathology 106:997-1005.

Churchill, A. C. L., Ciuffetti, L. M., Hansen, D. R., Van Etten, H. D., and Van Alfen, N. K. 1990. Transformation of the fungal pathogen Cryphonectria parasitica with a variety of heterologous plasmids. Curr. Genet. 17:25-31.

Feng, H. Q., Li, G. H., Du, S. W., Yang, S., Li, X. Q., de Figueiredo, P., and Qin, Q. M. 2017. The septin protein Sep4 facilitates host infection by plant fungal pathogens via mediating initiation of infection structure formation. Environ. Microbiol. 19:1730-1749.

Fillinger, S., Ruijter, G., Tamás, M. J., Visser, J., Thevelein, J. M., and d'Enfert, C. 2001. Molecular and physiological characterization of the NAD-dependent glycerol 3-phosphate dehydrogenase in the filamentous fungus Aspergillus nidulans. Mol. Microbiol. 39:145-157.

Fulbright, D. W. 1984. Effect of eliminating dsRNA in hypovirulent Endothia parasitica. Genetics 74:722-724.

Goswami, R. S. 2012. Targeted gene replacement in fungi using a splitmarker approach. Pages 255-269 in: Plant Fungal Pathogens. Methods in Molecular Biology (Methods and Protocols), Vol. 835. M. Bolton and B. Thomma, eds. Humana Press, New York.

Henikoff, S., and Henikoff, J. G. 1992. Amino acid substitution matrices from protein blocks. Proc. Natl. Acad. Sci. U.S.A. 89:10915-10919.

Hernández-Rodríguez, Y., Hastings, S., and Momany, M. 2012. The septin AspB in Aspergillus nidulans forms bars and filaments and plays roles in growth emergence and conidiation. Eukaryot. Cell 11:311-323. 
Juvvadi, P. R., Fortwendel, J. R., Rogg, L. E., Burns, K. A., Randell, S. H., and Steinbach, W. J. 2011. Localization and activity of the calcineurin catalytic and regulatory subunit complex at the septum is essential for hyphal elongation and proper septation in Aspergillus fumigatus. Mol. Microbiol. 82:1235-1259.

Kazmierczak, P., Kim, D. H., Turina, M., and Van Alfen, N. K. 2005. A hydrophobin of the chestnut blight fungus, Cryphonectria parasitica, is required for stromal pustule eruption. Eukaryot. Cell 4:931-936.

Kim, D. H., Rigling, D., Zhang, L., and Van Alfen, N. K. 1995. A new extracellular laccase of Cryphonectria parasitica is revealed by deletion of Lac1. Mol. Plant-Microbe Interact. 8:259-266.

Kim, J. M., Lee, J. G., Yun, S. H., So, K. K., Ko, Y. H., Kim, Y. H., Park, S. M., and Kim, D. H. 2016. A mutant of the Bckl homolog from Cryphonectria parasitica resulted in sectorization with an impaired pathogenicity. Mol. Plant-Microbe Interact. 29:268-276.

Kim, J. M., Park, J. A., and Kim, D. H. 2012. Comparative proteomic analysis of chestnut blight fungus, Cryphonectria parasitica, under tannic-acid-inducing and hypovirus-regulating conditions. Can. J. Microbiol. 58:863-871.

Kim, K. W., Park, E. W., Kim, Y. H., Ahn, K. K., Kim, P. G., and Kim, K. S. 2001. Latency- and defense-related ultrastructural characteristics of apple fruit tissues infected with Botryosphaeria dothidea. Phytopathology 91:165-172.

Kim, M. J., Park, S. M., Kim, Y. H., Cha, B. J., Yang, M. S., and Kim, D. H. 2004. Deletion of a hypoviral-regulated cppkl gene in a chestnut blight fungus, Cryphonectria parasitica, results in microcolonies. Fungal Genet. Biol. 41:482-492.

Kozubowski, L., and Heitman, J. 2010. Septins enforce morphogenetic events during sexual reproduction and contribute to virulence of Cryptococcus neoformans. Mol. Microbiol. 75:658-675.

Lee, J. K., Tattar, T. A., Berman, P. M., and Mount, M. S. 1992. A rapid method for testing the virulence of Cryphonectria parasitica using excised bark and wood of American chestnut. Phytopathology 82: 1454-1456.

Li, S., Myung, K., Guse, D., Donkin, B., Proctor, R. H., Grayburn, W. S., and Calvo, A. M. 2006. FvVE1 regulates filamentous growth, the ratio of microconidia to macroconidia and cell wall formation in Fusarium verticillioides. Mol. Microbiol. 62:1418-1432.

Lindsey, R., Cowden, S., Hernández-Rodríguez, Y., and Momany, M. 2010. Septins AspA and AspC are important for normal development and limit the emergence of new growth foci in the multicellular fungus Aspergillus nidulans. Eukaryot. Cell 9:155-163.

Livak, K. J., and Schmittgen, T. D. 2001. Analysis of relative gene expression data using real-time quantitative PCR and the $2^{-\Delta \Delta C T}$ method. Methods 25:402-408

Longtine, M. S., Theesfeld, C. L., McMillan, J. N., Weaver, E., Pringle, J. R., and Lew, D. J. 2000. Septin-dependent assembly of a cell cycleregulatory module in Saccharomyces cerevisiae. Mol. Cell. Biol. 20: 4049-4061.

Momany, M., Zhao, J., Lindsey, R., and Westfall, P. J. 2001. Characterization of the Aspergillus nidulans septin (asp) gene family. Genetics 157:969-977.

Nguyen, Q. B., Kadotani, N., Kasahara, S., Tosa, Y., Mayama, S., and Nakayashiki, H. 2008. Systematic functional analysis of calciumsignalling proteins in the genome of the rice-blast fungus, Magnaporthe oryzae, using a high-throughput RNA-silencing system. Mol. Microbiol. 68:1348-1365.

Nishihama, R., Onishi, M., and Pringle, J. R. 2011. New insights into the phylogenetic distribution and evolutionary origins of the septins. Biol. Chem. 392:681-687.

Nuss, D. L. 1992. Biological control of chestnut blight: An example of virus-mediated attenuation of fungal pathogenesis. Microbiol. Rev. 56: 561-576.

Pan, F., Malmberg, R. L., and Momany, M. 2007. Analysis of septins across kingdoms reveals orthology and new motifs. BMC Evol. Biol. 7:103.

Park, J. A., Kim, J. M., Park, S. M., and Kim, D. H. 2012. Characterization of CpSte11, a MAPKKK gene of Cryphonectria parasitica, and initial evidence of its involvement in the pheromone response pathway. Mol. Plant Pathol. 13:240-250.

Powell, W. A. J., Jr., and Van Alfen, N. K. 1987. Two nonhomologus viruses of Cryphonectria (Endothia) parasitica reduce accumulation of specific virulence-associated polypeptides. J. Bacteriol. 169: 5324-5326.

Puhalla, J. E., and Anagnostakis, S. L. 1971. Genetics and nutritional requirements of Endothia parasitica. Phytopathology 61:169-173.

Sheppard, D. C., Doedt, T., Chiang, L. Y., Kim, H. S., Chen, D., Nierman, W. C., and Filler, S. G. 2005. The Aspergillus fumigatus StuA protein governs the up-regulation of a discrete transcriptional program during the acquisition of developmental competence. Mol. Biol. Cell 16: 5866-5879.

Van Alfen, N. K. 1982. Biology and potential for disease control of hypovirulence of Endothia parasitica. Annu. Rev. Phytopathol. 20: 349-362.

Van Alfen, N. K., Jaynes, R. A., Anagnostakis, S. L., and Day, P. R. 1975. Chestnut blight: Biological control by transmissible hypovirulence in Endothia parasitica. Science 189:890-891.

Vargas-Muñiz, J. M., Juvvadi, P. R., and Steinbach, W. J. 2016. Forging the ring: From fungal septins' divergent roles in morphology, septation and virulence to factors contributing to their assembly into higher order structures. Microbiology 162:1527-1534.

Vargas-Muñiz, J. M., Renshaw, H., Richards, A. D., Lamoth, F., Soderblom, E. J., Moseley, M. A., Juvvadi, P. R., and Steinbach, W. J. 2015. The Aspergillus fumigatus septins play pleiotropic roles in septation, conidiation, and cell wall stress, but are dispensable for virulence. Fungal Genet. Biol. 81:41-51.

Versele, M., Gullbrand, B., Shulewitz, M. J., Cid, V. J., Bahmanyar, S., Chen, R. E., Barth, P., Alber, T., and Thorner, J. 2004. Protein-protein interactions governing septin heteropentamer assembly and septin filament organization in Saccharomyces cerevisiae. Mol. Biol. Cell 15:4568-4583.

Versele, M., and Thorner, J. 2005. Some assembly required: Yeast septins provide the instruction manual. Trends Cell Biol. 15:414-424.

Warenda, A. J., Kauffman, S., Sherrill, T. P., Becker, J. M., and Konopka, J. B. 2003. Candida albicans septin mutants are defective for invasive growth and virulence. Infect. Immun. 71:4045-4051.

\section{AUTHOR-RECOMMENDED INTERNET RESOURCE}

C. parasitica genome sequence information: http://genome.jgi-psf.org/Crypa2/Crypa2.home.html 